\title{
Peritoneal Dialysis Penetration and Peritonitis Rate at a Single Centre during Last Decade
}

\author{
Jana Uhlinova, Ülle Pechter, Kadri Kermes, and Mai Ots-Rosenberg \\ Department of Internal Medicine, University of Tartu, 6 Puusepa Street, Tartu 51014, Estonia \\ Correspondence should be addressed to Mai Ots-Rosenberg, mai.ots@kliinikum.ee
}

Received 31 January 2011; Revised 6 April 2011; Accepted 19 April 2011

Academic Editor: Paul Tam

Copyright (c) 2011 Jana Uhlinova et al. This is an open access article distributed under the Creative Commons Attribution License, which permits unrestricted use, distribution, and reproduction in any medium, provided the original work is properly cited.

\begin{abstract}
Peritoneal dialysis (PD) has been intensively offered at our centre to patients (pts) with end-stage renal disease (ESRD) from 2000 , and the number of PD pts was noticed to raise. We aimed to analyse the PD population from the aspect of penetration and peritonitis rate during eleven years. Cumulative number of new RRT pts was 378 during the study period. We found high PD penetration rate: 53\% (range 32-72\%). The rate of peritonitis was as high as 9.8 during first study years, but it has declined progressively last year being 29.1 by September 2010 and 21.7 by December 2010. Most cases of peritonitis were due to grampositive pathogens. We have demonstrated steady high single-centre PD penetration rate and improvement of management of patients during last decade probably because of the result of better pts education and a continued dedication of the staff.
\end{abstract}

\section{Introduction}

Penetration of peritoneal dialysis varies widely across the world. It ranges from about $80 \%$ in Hong Kong and Mexico to few percentage points in the United States and some developing countries [1-5]. Peritoneal dialysis appears to have some excellent properties as a first-line renal replacement therapy (RRT) [6]. The use of dialysis and transplantation as complementary therapies for RRT is well established in our country. RRT data at December 31 , each year have been reported regularly to European Dialysis and Transplantation Association Registry [7] and detailed epidemiologic data available in Annual Report of Kidney Diseases in Estonia 2009 [8]. According to reports, the incidence and prevalence of RRT patients in our country remains lower than that reported from the other European countries. The incidence of RRT has a decreasing tendency during last three years in the country being $62.7 \mathrm{pmp}$ at day 91 in 2008 [7]. Peritoneal dialysis (PD) was introduced to the clinical practice already 17 years ago at our university hospital which is the second largest centre in the country. Since 2000, after structural changes at our centre when nephrology division was connected with dialysis unit, we started to intensively offer PD treatment to every patient with end-stage renal disease (ESRD) without contraindications for $\mathrm{PD}$, and the number of $\mathrm{PD}$ patients raised sharply. The number of peritoneal dialysis patients have been the highest in the country compared with other centres [8]. According to the Annual Report PD, patients formed 47\% from dialysis prevalence patients at Tartu University Hospital at the end of 2009, whereas the percentage was lower in other centres: $21 \%$ at West-Tallinn Hospital and 44\% at North-Estonia Regional Hospital [8]. Peritonitis remains a significant problem in peritoneal dialysis. It is the leading cause of technique failure in peritoneal dialysis. Therefore, we aimed to analyse our PD population in a single-centre cohort study with respect to penetration and peritonitis rate during last decade.

\section{Materials and Methods}

The report is based on retrospective data from patient's records, and comparable whole country data were obtained from Annual Report of Kidney Diseases 2009. PD penetration rate was defined as the percentage of new patients on $\mathrm{PD}$ in relation to all new dialysis patients each year. PD penetration rate, peritonitis rate, and microbiology of peritoneal fluid have been analysed for all pts in our program since 2000. In conjunction with epidemiological research study in the country, we recently expanded the 
data set where individual RRT patients data together with clinical performance indicators (CPI) at the end of each year have been collected and analyzed beside the basic RRT epidemiological data collection. In the current investigation, we demonstrate our single centre PD patients CPIs that characterize anaemia, calcium phosphate, and lipids mean patients group levels. The following biochemical parameters (using the Hitachi 912 Analyzer until 2004 and COBAS INTEGRA 800 after 204) were studied: serum creatinine (S-Crea, $\mu \mathrm{mol} / \mathrm{L}$ ), serum albumin (S-Alb, g/L), C-reactive protein (CRP, mg/L), serum-ionized calcium (Si-Ca, mmol/l), serum total calcium (S-total $\mathrm{Ca}, \mathrm{mmol} / \mathrm{L}$ ), and serum phosphate (S-P, mmol/L). Studied lipid profile was the following: serum total cholesterol (S-Chol, $\mathrm{mmol} / \mathrm{L}$ ), serum HDL cholesterol (S-HDL chol, mmol/L), serum LDL cholesterol (S-LDL chol, $\mathrm{mmol} / \mathrm{L}$ ), and serum triglyceride (TG, mmol/L). Parathyroid hormone (PTH, pmol/L) levels were determined by immunoanalyzer IMMULITE 2000 using chemiluminescence method. Haemoglobin ( $\mathrm{Hgb} g / \mathrm{L})$ levels were measured by photometric method. Peritonitis was defined as turbid fresh dialysis effluent containing polynuclear leukocyte cell count higher than $100 / \mathrm{mm}^{3}$. Peritonitis rate was calculated as number of peritonitis episodes per number of patients-months. For the isolation of the organisms, blood culture media was used (BACTEC Microbiological Culture Analyzer). Two thirds of patients were on Baxter DUO connection system, and one third of patients remained on Fresenius stay-safe system.

\section{Results and Discussion}

The retrospective study was carried out at the Department of Internal Medicine of Tartu University. Cumulative number of new RRT patients in our centre who started peritoneal dialysis between January 2000 and December 2010 was 378 . Mean age of all incidence dialysis patients was 58.8 years in 2010 with male predominance of $57 \%$. Demographic data are comparable with country RRT incidence data showed in Annual Report of Kidney Diseases 2009. According to the Report, the mean age of incidence patients was 60.5 years, percentage of males $58 \%$. Diabetes is the main cause of ESRD in new dialysis patients in Estonia [8]. Table 1 shows the main causes of ESRD patients at our centre and comparable data of whole country. Because of small numbers, our centre incidence patients diabetes diagnosis percentage differs from year to year being still high every year.

Figure 1 showed the percentage of mean peritoneal dialysis penetration rate which was 53\% (range 32-72\%) in our centre during the eleven year-study period. With these results, we demonstrate a steady peritoneal dialysis penetration rate during long period. In our opinion, this is because of better patients education and a continued dedication of the staff. It is important that patients and nurses are well educated in the practice of peritoneal dialysis. Similarly, in other long-term studies, many centres report higher penetration rate after essential improvement of local skills [1-3].

Clinical and laboratory data are demonstrated in Table 2. Results show that many of our peritoneal dialysis patients

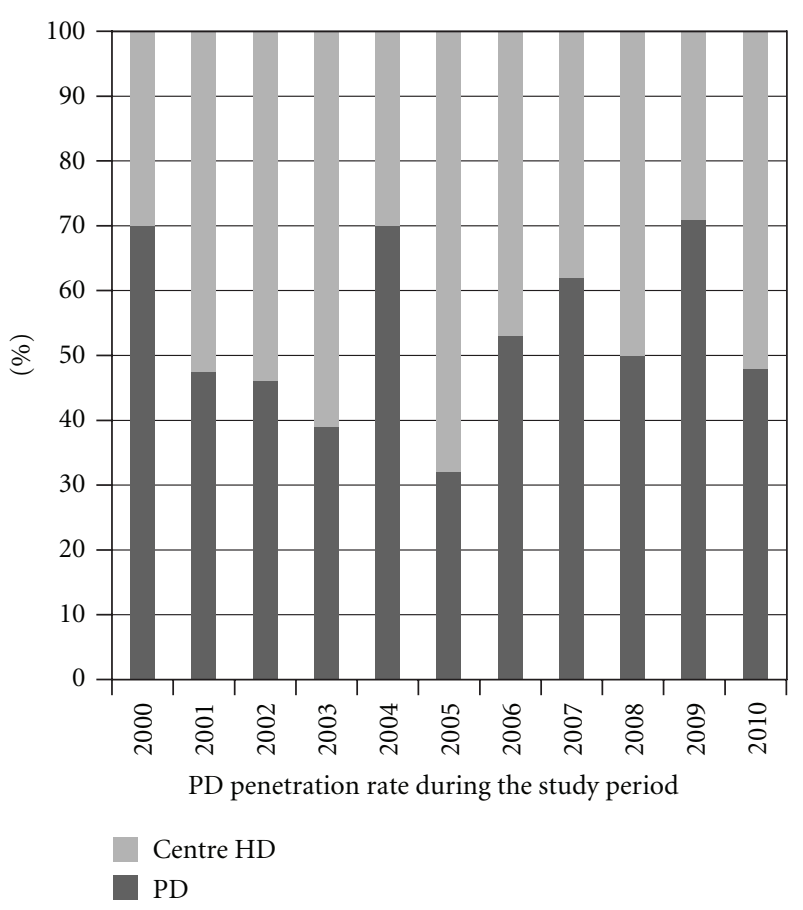

FIGURE 1: Peritoneal dialysis penetration rate at the Tartu University Hospital 2000-2010.

have overweight, increased inflammatory status, hypoalbuminemia and dyslipidemia. Inflammation and dyslipidemia accelerate atherosclerosis and are often present in peritoneal dialysis patients $[9,10]$. Normal range of haemoglobin according to guidelines [11] was found in our study patients.

Arterial calcification is common in adults with chronic kidney disease and progresses with time. On the other hand, peritoneal calcification, one of the major complications, can develop in peritoneal dialysis patients [12]. Therefore, the management of secondary hyperparathyroidism and avoidance of peritonitis should be important aims of the treatment. We found that, although, calcium and phosphate levels were almost in normal range, mean PTH was increased in our patients (Table 2). We conclude that further improvement of the management of mineral metabolism and secondary hyperparathyroidism as well as body composition measurements and calcification diagnostics are needed at our centre to avoid serious complications described by many authors [12-15].

Peritonitis remains a major complication in patients undergoing peritoneal dialysis and remains a major cause of patients discontinuing peritoneal dialysis and switching to haemodialysis. However, technique survival at year 1 has been almost $100 \%$ at our center during last years. Because of short period and small numbers survival, data are not included and will be shown separately. Our study analyzed peritonitis rate and pathogens responsible for the peritonitis. The incidence of peritoneal dialysis-related peritonitis was as high as 1 episode every 9.8 months in 2004, but it has progressively declined during the last years being 29.1 in September 2010 and 21.7 at the end of the year (Table 3 ). 
TABLe 1: Aetiology of chronic kidney disease in dialysis incident and prevalent patients at Tartu University Hospital during 2008-2010 and in Estonia in 2009.

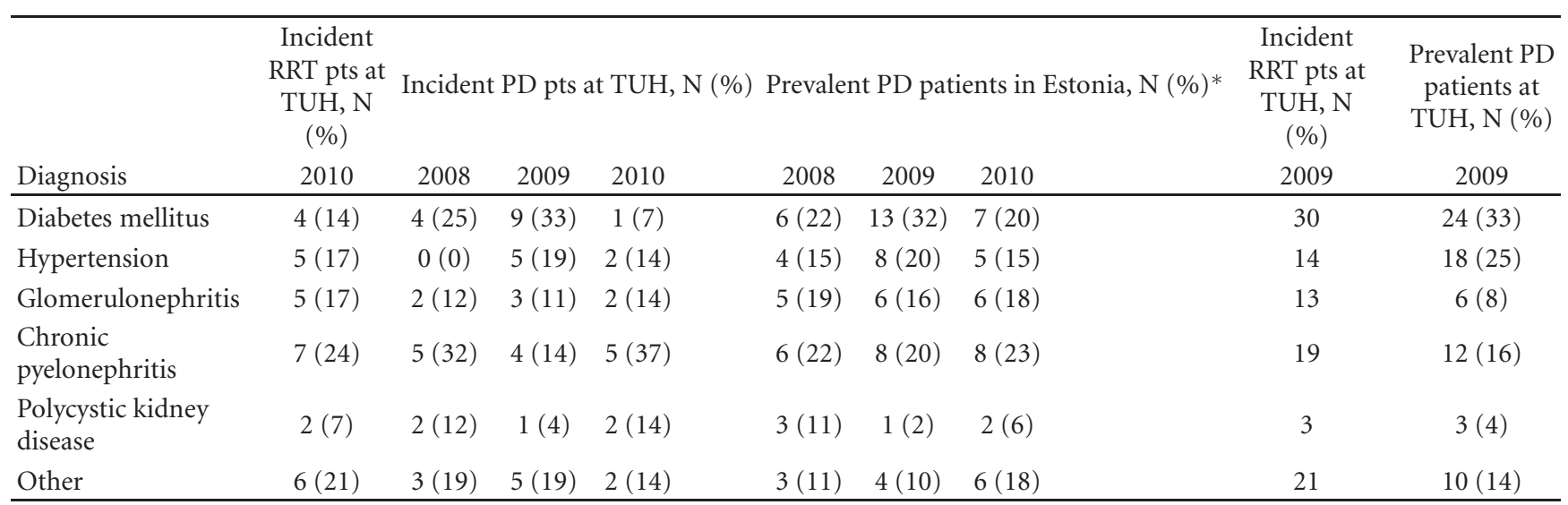

* Data from Annual Report of Kidney Diseases in Estonia 2009.

Abbreviations: TUH, Tartu University Hospital; RRT, renal replacement therapy; PD, peritoneal dialysis; N, number.

TABLE 2: Clinical and laboratory mean data in prevalent peritoneal dialysis patients group at the end of year 2010 .

\begin{tabular}{lcccc}
\hline Variables & PD pts group mean & SD $^{*}$ & Min & Max \\
\hline Patients total $\mathbf{n}=\mathbf{3 4}$ & 28.8 & 1.1 & 19 & 43.6 \\
\hline BMI $\left(\mathrm{kg} / \mathrm{m}^{2}\right)$ & 113.4 & 2.6 & 94 & 157 \\
Haemoglobin $(\mathrm{g} / \mathrm{L})$ & 705.5 & 45.6 & 316 & 1112 \\
S-creatinine $(\mu \mathrm{l} / \mathrm{L})$ & 34.3 & 0.9 & 19 & 42 \\
S-albumin $(\mathrm{g} / \mathrm{L})$ & 9.5 & 1.5 & 1 & 32 \\
C-reactive protein $(\mathrm{mg} / \mathrm{L})$ & 1.2 & 0.0 & 1.0. & 1.4 \\
S-ionized calcium $(\mathrm{mmol} / \mathrm{L})$ & 2.4 & 0.0 & 1.7 & 2.8 \\
S-total calcium $(\mathrm{mmol} / \mathrm{L})$ & 1.8 & 0.1 & 0.6 & 4.4 \\
S-phosphate $(\mathrm{mmol} / \mathrm{L})$ & 52.2 & 10.9 & 1.6 & 197 \\
PTH (pmol/L) & 5.7 & 0.2 & 3.0 & 7.8 \\
S-total cholesterol $(\mathrm{mmol} / \mathrm{L})$ & 1.2 & 0.1 & 0.6 & 1.8 \\
S-HDL cholesterol $(\mathrm{mmol} / \mathrm{L})$ & 3.9 & 0.2 & 1.9 & 6.2 \\
S-LDL cholesterol $(\mathrm{mmol} / \mathrm{L})$ & 1.9 & 0.2 & 0.8 & 5.4 \\
S-triglycerides $(\mathrm{mmol} / \mathrm{L})$ & & & &
\end{tabular}

${ }^{*}$ SD- standard deviation.

The improvement that we noticed between 2004 and 2006 may be the result of change in the connection systems at that time. Currently, during last years, almost two thirds of patients were on Baxter DUO connection system and one third of patients remained on Fresenius stay-safe system.

Many centres have been reported that, over time, the microbiology at those institutions has been changing [16]. We cannot confirm this because the aetiology of peritonitis have been similar many years. Table 4 demonstrates that coagulase-negative staphylococcus has been the most common pathogen during 2006-2010 followed by Staphylococcus aureus. In two effluents in 2009 and three in 2010, more than one pathogen was isolated. Thus, most cases of peritonitis were due to Gram-positive pathogens, accounting around $35 \%$ of all peritonitis episodes, and Gram-negative infections were presented with a variety of different organisms, predominantly E. coli.
TABle 3: Peritonitis rate at Tartu University Hospital.

\begin{tabular}{|c|c|c|c|c|c|}
\hline Year & 2004 & 2006 & 2008 & $2010^{*}$ & 2010 \\
\hline Pts total $\mathrm{nr}$ & 42 & 46 & 45 & 42 & 45 \\
\hline $\begin{array}{l}\text { Pts nr at the end of the } \\
\text { year }\end{array}$ & 29 & 34 & 27 & 35 & 34 \\
\hline Treatment months & 354 & 303 & 409 & 408 & 500 \\
\hline Peritonitis nr & 36 & 19 & 19 & 14 & 23 \\
\hline $\begin{array}{l}\text { Peritonitis rate } \\
\text { (episodes/nr } \\
\text { pts-months) }\end{array}$ & 9.8 & 16 & 21.5 & 29.1 & 21.7 \\
\hline $\begin{array}{l}\text { Peritonitis rate } \\
\text { (episodes/pts-year) }\end{array}$ & 1.2 & 1.3 & 1.8 & 2.4 & 1.8 \\
\hline
\end{tabular}

* January-September 2010.

TABLE 4: Etiology of peritonitis at Tartu University Hospital.

\begin{tabular}{lcc}
\hline Pathogen & $\begin{array}{c}2009-2010, \\
\mathrm{~N}(\%)\end{array}$ & $\begin{array}{c}2006-2007, \\
\mathrm{~N}(\%)\end{array}$ \\
\hline Coagulase-negative staphylococci & 21 & 15 \\
Staphylococcus aureus & 11 & 10 \\
Streptococci & 2 & 6 \\
Gram-positive rod-shaped bacteriae & 5 & 3 \\
Enterococci & 4 & 2 \\
Enterobacteria & 7 & 3 \\
Acinetobacter baumannii & 1 & 0 \\
Pseudomonas aeruginosa & 0 & 1 \\
Yeasts & $2(3 \%)$ & 0 \\
\hline Gram-positive organisms & $43(74 \%)$ & $36(84 \%)$ \\
Gram-negative organisms & $8(14 \%)$ & $4(9 \%)$ \\
Culture-negative peritonitis & $5(9 \%)$ & $3(7 \%)$ \\
\hline Total & $58(100 \%)$ & $43(100 \%)$ \\
\hline
\end{tabular}

\section{Conclusions}

This is a first report on long-term peritoneal dialysis experience at a single centre in Estonia. We have demonstrated 
steady high single-centre peritoneal dialysis penetration rate and declining tendency of peritonitis rate after essential improvement of local skills.

\section{Acknowledgments}

The authors thank Maie Pikkmaa, Pirgit Palk, Siiri Mesikepp, Elviira Seppet, Eino Sinimäe, Külli Kõlvald, and Kristi Veermäe.

\section{References}

[1] H. J. Gloor, S. Pandolfi, and S. Rüttimann, "20 years of peritoneal dialysis in a mid-sized Swiss hospital," Swiss Medical Weekly, vol. 133, no. 45-46, pp. 619-624, 2003.

[2] B. L. Goh, Y. M. Ganeshadeva, S. E. Chew, and M. S. Dalimi, "Does peritoneal dialysis catheter insertion by interventional nephrologists enhance peritoneal dialysis penetration?" Seminars in Dialysis, vol. 21, no. 6, pp. 561-566, 2008.

[3] W. K. Lo, "Peritoneal dialysis utilization and outcome: what are we facing?" Peritoneal Dialysis International, vol. 27, no. 2, pp. S42-S47, 2007.

[4] R. Mehrotra, "Peritoneal dialysis penetration in the United States: march toward the fringes?" Peritoneal Dialysis International, vol. 26, no. 4, pp. 419-422, 2006.

[5] I. Najafi et al., "The story of continuous ambulatory peritoneal dialysis in Iran," International Society for Peritoneal Dialysis, vol. 30, no. 4, pp. 430-433, 2010.

[6] W. Van Biesen, "Peritoneal dialysis: how we can achieve improvement of PD penetration," Prilozi, vol. 28, no. 1, pp. 267-274, 2007.

[7] Registry, "ERA-EDTA," Registry Annual Report, 2010, http:// www.era-edta-reg.org/files/annualreports/pdf/AnnRep2008 .pdf.

[8] M. Rosenberg et al., "Epidemiological Data of Kidney Diseases in Estonia," Annual Report 2009, pp. 1-16, 2010, http://www .nephro-quest.eu/files/deliverable/D15\%20-\%206.4\%20WP6 \%20TU\%20Estonia\%20Annual\%20Registry\%20Report $\% 202009$.pdf.

[9] O. Heimbürger, "Lipid disorders, statins and the peritoneal membrane," Contributions to Nephrology, vol. 163, pp. 177$182,2009$.

[10] M. Kon et al., "Profiles of inflammatory markers and lipoprotein subclasses in patients undergoing continuous ambulatory peritoneal dialysis," Clinica Chimica Acta, vol. 411, no. 21-22, pp. 1723-1727, 2010.

[11] F. Locatelli, A. Covic, K. U. Eckardt, A. Wiecek, and R. Vanholder, "Anaemia management in patients with chronic kidney disease: a position statement by the Anaemia Working Group of European Renal Best Practice (ERBP)," Nephrology Dialysis Transplantation, vol. 24, no. 2, pp. 348-354, 2009.

[12] A. Vlijm et al., "Are peritoneal calcifications in long-term peritoneal dialysis related to aortic calcifications and disturbances in mineral metabolism?" Nephrology Dialysis Transplantation, vol. 26, no. 1, pp. 304-308, 2010.

[13] S. M. Deger et al., "Can calcium, phosphate, calcium phosphate product and intact parathyroid hormone levels be appropriately controlled in dialysis patients?" Medical Principles and Practice, vol. 20, no. 1, pp. 85-89, 2010.

[14] K. Trigka, P. Dousdampanis, M. Chu et al., "Encapsulating peritoneal sclerosis: a single-center experience and review of the literature," International Urology and Nephrology, vol. 43, no. 2, pp. 519-526, 2011.

[15] A. De Araujo Antunes, F. D. Vannini, L. C. Martin et al., "Inflammation and overweight in peritoneal dialysis: is there an association," Renal Failure, vol. 31, no. 7, pp. 549-554, 2009.

[16] S. B. Furgeson and I. Teitelbaum, "New treatment options and protocols for peritoneal dialysis-related peritonitis," Contributions to Nephrology, vol. 163, pp. 169-176, 2009. 


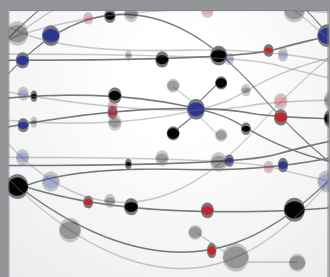

The Scientific World Journal
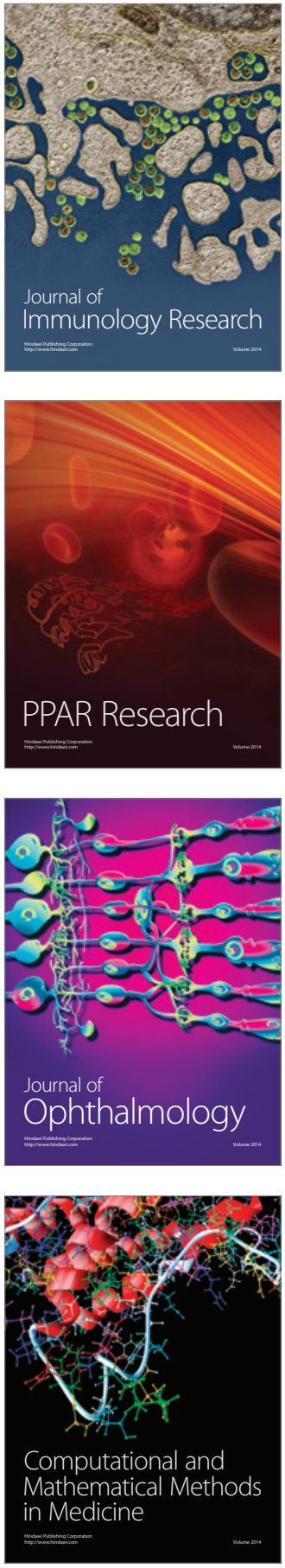

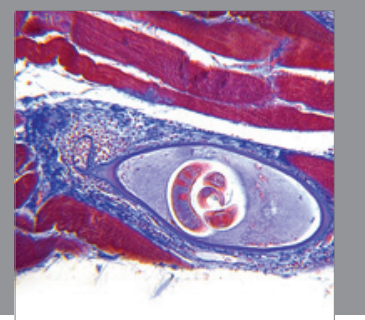

Gastroenterology

Research and Practice
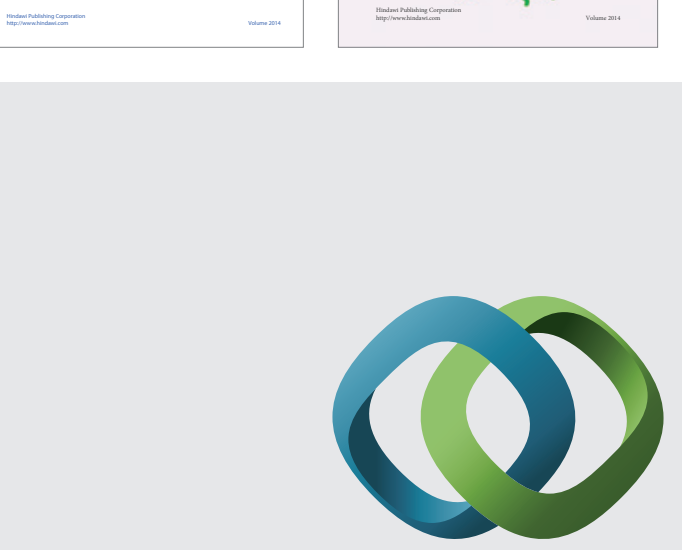

\section{Hindawi}

Submit your manuscripts at

http://www.hindawi.com
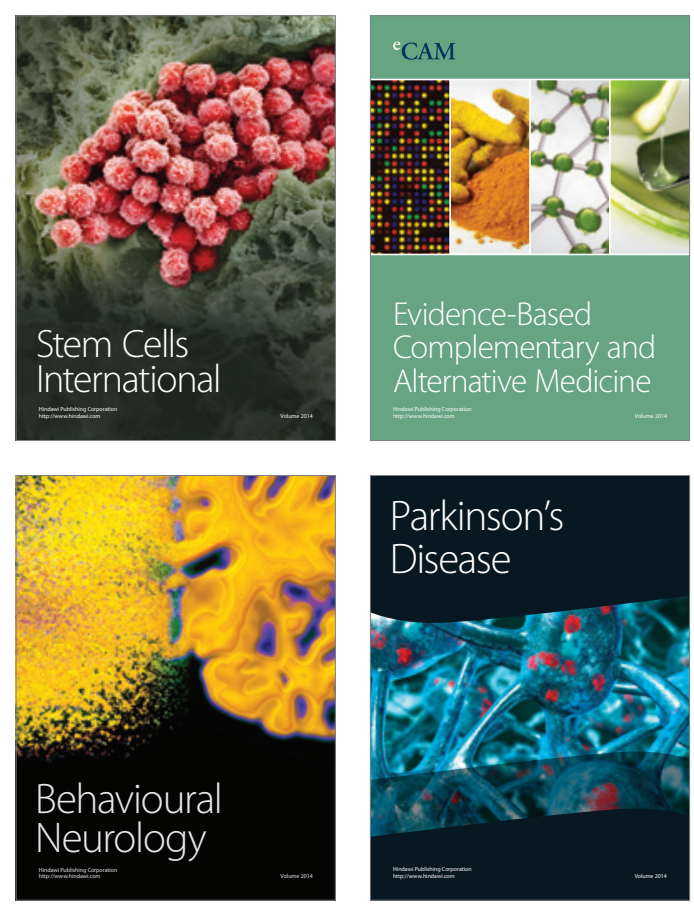

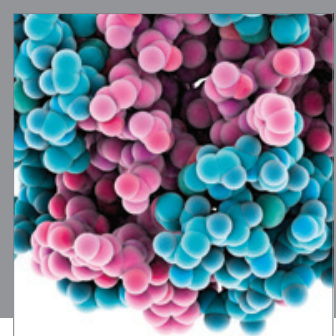

Journal of
Diabetes Research

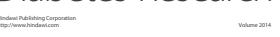

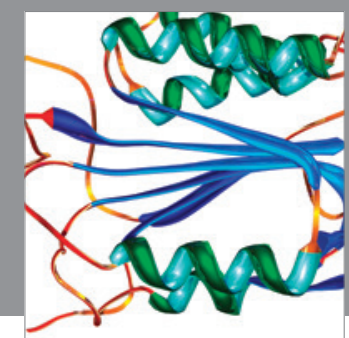

Disease Markers
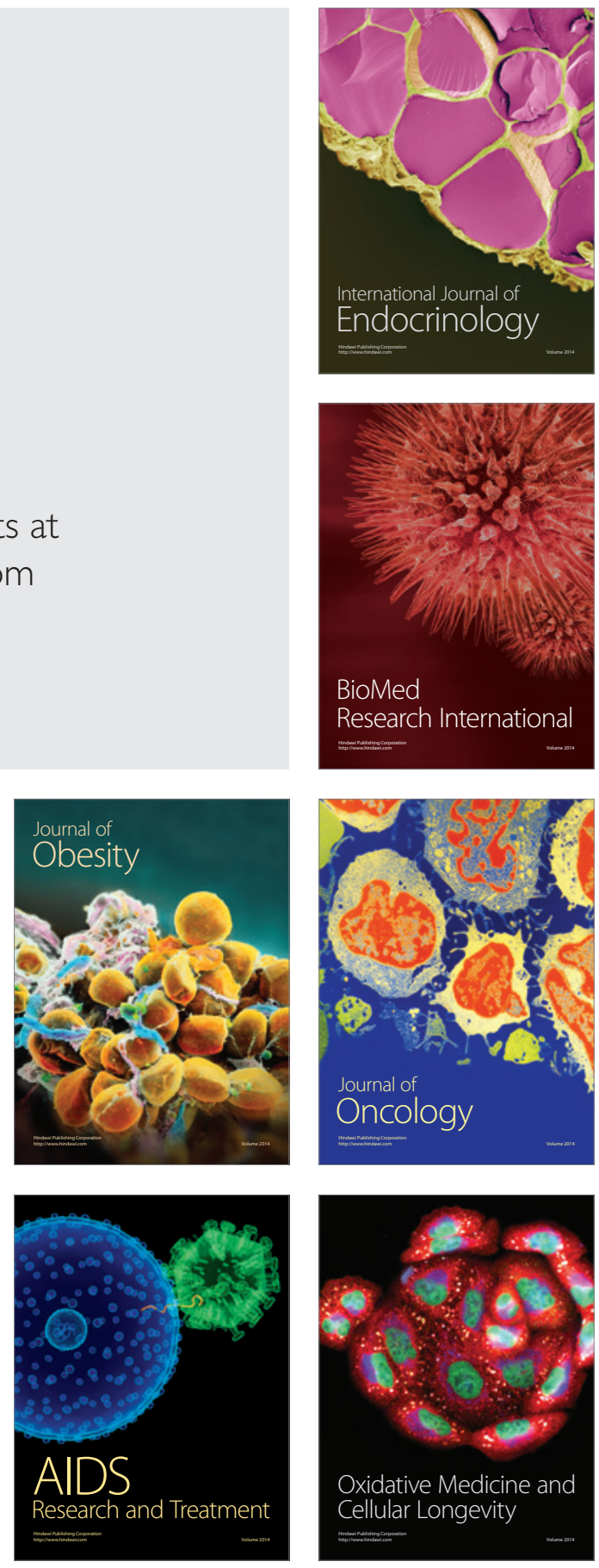